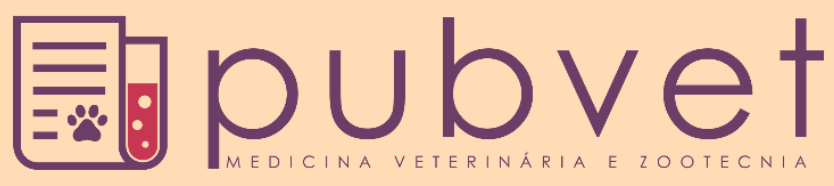

https://doi.org/10.31533/pubvet.v15n12a991.1-9

\title{
Bem-estar de bovinos leiteiros acometidos por afecções digitais como evidência nas perícias veterinárias: Revisão
}

\author{
Jackson Barros do Amaral ${ }^{1 * \bullet}$, Vinícius José Moreira Nogueira ${ }^{2 \bullet}$ \\ ${ }^{I}$ Pesquisador Científico, Médico Veterinário, Secretaria de Agricultura e Abastecimento-SAA do Estado de São Paulo, Instituto de Zootecnia, \\ Centro de Pesquisa de Bovinos Leite, Brasil. \\ ${ }^{2}$ Médico Veterinário, Professor da Fundação de Ensino e Pesquisa do Sul de Minas Gerais (FEPESMIG), Curso Medicina Veterinária do \\ Centro Universitário do Sul de Minas (UNIS), Brasil. \\ *Autor para correspondência, E-mail: jackson.amaral@sp.gov.br
}

Resumo. As afecções digitais dos bovinos leiteiros estão frequentemente associadas a dor e claudicação e causam grande impacto no bem-estar animal. Nesta espécie animal o bemestar constitui uma preocupação mundial e vem sendo debatido do ponto de vista econômico, científico, social e humanitário. As perícias apresentam destaque fundamental para conhecer graus de bem-estar, assim como constatar condições de negligências, imprudência ou imperícia em casos maus-tratos, associados ou não a condutas dolosas do agente. A dor está presente em todos os sistemas de exploração de bovinos leiteiros e as afecções digitais representam importante problema de saúde que compromete o bem-estar. A clínica médica forense aplicada aos bovinos permite diagnosticar ou suspeitar de casos de afecções digitais que não estão relacionadas às contingências de erros de manejo, instalações inadequadas, condições despercebidas ou até ocultadas pelos proprietários e funcionários, reveladas pelos médicos veterinários apenas durante os exames clínicos de rotina. As afecções digitais em bovinos leiteiros são afecções multifatoriais que envolvem os animais, o manejo, a saúde e o ambiente de criação do rebanho e tem como causas as atitudes e decisões do pecuarista na prevenção e tratamento destas patologias e nas mudanças de atitudes dos mesmos visando um novo paradigma de produção. A negligência, a imperícia e a imprudência podem afetar os bovinos por maus-tratos, crueldade ou abuso. As evidências produzidas por exames clínico e diagnóstico de bemestar de bovinos acometidos por afecções digitais são fundamentais para produção de provas nas perícias veterinárias. A constatação de fatores de riscos envolvidos nas afecções digitais tem importância fundamental para a dimensão de um novo um paradigma de exploração de bovinos leiteiros. As decisões judiciais em casos de maus-tratos podem ser subsidiadas por pareceres técnicos de especialistas em bem-estar animal. Casos de maustratos, crueldade ou abuso por negligência imperícia e imprudência podem ser constatados pelas perícias de bem-estar em laudos utilizados em questões judiciais. Esta revisão tem o objetivo de discutir aspectos das afecções digitais em bovinos leiteiros pela revisão de natureza bibliográfica com ênfase nas evidências do diagnóstico de bem-estar para elaboração de laudos periciais nos casos suspeitos de maus-tratos por negligência, imperícia e/ou imprudência.

Palavras chave: Bovinocultura, claudicação, medicina veterinária legal, peritagem, podopatias

\section{Welfare of dairy cattle suffering from digital disorders as evidence in veterinary investigation: Revision}

Abstract. Digital disorders of dairy cattle are frequently linked to pain and lameness and cause a great impact on animal welfare. Animal welfare is a world concern, especially for this species in particular, and it has been discussed from economic, scientific, social and humanitarian 
perspectives. Veterinarian investigations are vital to understand the animal welfare levels, as well as to verify neglectful conditions, imprudence or unskillfulness or even abuse, whether or not intentional. Pain is present in all dairy cattle farming systems and digital disorders represent a major health problem, which compromises well-being. Forensics applied to cattle enables diagnosis or suspicion of digital conditions that are not related to the contingencies of livestock handling errors, inappropriate facilities, unnoticed conditions or even restricted by the owners and employees, disclosed by veterinarian doctors only through routine clinical examinations. Digital disorders in dairy cattle are multifactorial conditions concerning animals, handling, health and environment, caused by farmers' actions and decisions related to preventing and treating such pathologies and in changing their attitudes towards a new production paradigm. Negligence, unskillfulness and imprudence may affect cattle due to mistreatment, cruelty or abuse. The material produced through clinical examination and diagnosis of cattle welfare for those animals with digital conditions are essential for the production of evidence in Veterinary investigation. Finding risk factors involved in digital conditions is of crucial importance, considering the aspects of a new paradigm in dairy cattle farming. Legal decisions in cases of animal abuse can be supported by technical opinions of experts on animal welfare. Cases of mistreatment, cruelty or abuse for neglect, unskillfulness and imprudence can be verified by the animal welfare investigation in reports used in legal issues. This review aims to discuss aspects of digital disorders in dairy cattle through a bibliographic review with emphasis on the material obtained in welfare diagnosis in order to prepare expert reports in suspected cases of animal abuse by negligence, unskillfulness and/or imprudence.

Keywords: Cattle farming, lameness, veterinary forensics, expert investigation, podopathies

\section{Bienestar del ganado lechero afectado por condiciones digitales como evidencia en la pericia veterinaria: Revisión}

Resumen. Los trastornos digitales del ganado lechero a menudo se asocian con dolor y cojera y tienen un gran impacto en el bienestar de los animales. En esta especie animal, el bienestar es una preocupación mundial y se ha debatido desde el punto de vista económico, científico, social y humanitario. La pericia es fundamental para conocer los grados de bienestar, así como para verificar las condiciones de negligencia, imprudencia o impericia en casos de maltrato, asociado o no con la conducta deliberada del agente. El dolor está presente en todos los sistemas de ganadería lechera y los trastornos digitales representan un importante problema de salud que compromete el bienestar. La clínica médica forense aplicada al ganado bovino permite diagnosticar o sospechar casos de afecciones digitales que no están relacionadas con contingencias de errores de gestión, instalaciones inadecuadas, condiciones desapercibidas o incluso ocultas por propietarios y empleados, revelados por veterinarios solo durante la rutina exámenes clínicos. Los trastornos digitales en el ganado lechero son afecciones multifactoriales que afectan a los animales, la gestión, la salud y el entorno de cría del rebaño y sus causas son las actitudes y decisiones de los ganaderos en la prevención y tratamiento de estas patologías y sus cambios en las actitudes, con el objetivo de crear un nuevo paradigma de producción. La negligencia, la impericia y la imprudencia pueden afectar al ganado bovino debido a malos tratos, crueldad o abuso. Las evidencias producidas por los exámenes clínicos y el diagnóstico del bienestar del ganado vacuno afectado por condiciones digitales es fundamental para la producción de pruebas en la pericia veterinaria. El hallazgo de los factores de riesgo implicados en las condiciones digitales reviste una importancia fundamental para el tamaño de un nuevo paradigma de explotación de ganado lechero. Las decisiones judiciales en casos de malos tratos pueden subvencionarse mediante el asesoramiento técnico de expertos en bienestar animal. Los casos de malos tratos, crueldad o abuso debidos a negligencia, imprudencia e impericia pueden verse en las aptitudes sociales en los informes utilizados en asuntos judiciales. Esta revisión tiene como objetivo discutir aspectos de los trastornos digitales del ganado lechero mediante una revisión bibliográfica con énfasis en la evidencia del diagnóstico de bienestar para la preparación de informes periciales en casos sospechosos de maltrato debido a negligencia, impericia e imprudencia.

Palabras claves: Ganadería, cojera, medicina veterinaria legal, opinión de pericia, podopatías 


\section{Introdução}

O bem-estar animal passou a ser considerado um dos critérios de sustentabilidade dos sistemas de produção (Koknaroglu \& Akunal, 2013). A qualidade dos produtos de origem animal é julgada em relação á ética na sua produção, incluindo os impactos causados ao meio ambiente e ao bem-estar dos animais (Broom, 1991; Broom \& Fraser, 2010). A dor está presente em todos os sistemas de exploração de bovinos leiteiros e as afecções digitais são as principais causas que comprometem a saúde, reprodução e bem-estar dos bovinos leiteiros que levam a prejuízos aos pecuaristas (Lean \& Golder, 2019). São afecções complexas, multifatoriais e envolvem os animais, ambiente, microrganismos e o homem, sendo o maior desafio minimizar os efeitos negativos para assegurar padrão tecnológico de produção (Amaral \& Trevisan, 2017; Luna, 2006). A frequência de problemas locomotores é mais alta em bovinos criados em sistemas de confinamento e semi-confinamento do que em animais criados extensivamente e a dor é uma característica comum nestas afecções (Ferreira et al., 2004; Ollhoff et al., 2005). A perícia médico-legal constitui um conjunto de procedimentos médicos e técnicos com objetivo esclarecer fatos de interesse da justiça, podendo também ser realizada em animais (Croce \& Croce Júnior, 2017; França, 2005). As decisões judiciais sobre casos de maus-tratos contra animais podem ser subsidiadas por laudos de peritos em bem-estar animal. Graus de bem-estar baixo ou muito baixo são inaceitáveis e devem ser descritos como maus-tratos em questões judiciais. A elucidação de maus-tratos, crueldade e abuso em animais tem sido considerada tema de relevância. Este artigo tem o objetivo de discutir aspectos das afecções digitais em bovinos leiteiros pela revisão de natureza bibliográfica com ênfase nas evidências do diagnóstico de bem-estar para elaboração de laudos periciais nos casos suspeitos de maus-tratos por negligência, imperícia e/ou imprudência.

\section{Afecções digitais em bovinos leiteiros}

A dor e o sofrimento estão presentes em todos os sistemas de exploração de bovinos leiteiros e as afecções digitais, associadas ou não às claudicações, representam um dos mais importantes problemas de saúde que comprometem o bem-estar desta categoria animal (Amaral \& Trevisan, 2017). As afecções podais causam também grandes prejuízos econômicos na produção leiteira. Em bovinos acometidos por afecções digitais a dor, o sofrimento e o desconforto são condições mórbidas que levam à redução da ingestão de alimentos resultando em perdas da condição corporal. Estes animais podem apresentar imunodepressão e comprometimento geral do seu estado, predispondo a outras patologias como mastite, metrite, desordens reprodutivas metabólicas (Garbarino et al., 2004; Souza et al., 2006).

As lesões de afecções digitais apresentam fatores de ordem multifatoriais que envolvem causas infecciosas, traumáticas ou ambientais (Silva et al., 2006). Dietas ricas em carboidratos, falta de cuidados em podologia preventiva com aparas e correções das estruturas dos cascos, manejo dos animais em pisos úmidos e terrenos pedregosos são fatores de riscos que contribuem para ocorrência e desenvolvimento de afecções digitais (Silva et al., 2016). Enfermidades metabólicas, tais como acidose láctea ruminal provocam laminites ou pododermatites assépticas com claudicação e dor em bovinos (Ollhoff \& Ortolani, 2001). Além disso, a laminite comumente está associada a ruminal, a genética, ao manejo inadequado da parturiente, conforto e instalações (Mülling et al., 2006).

Animais de produção estão frequentemente expostos a uma rotina que pode leva-los a dor ou desconforto como em procedimentos de castração, descorna, auxílios obstétricos quando realizados de maneira errônea, sem aplicação prévia de anestesia e analgesia (Hudson et al., 2008; Luna, 2006) (Hudson et al., 2008; Luna, 2008). As claudicações em bovinos estão frequentemente associadas a dor que produzem desordens biomecânicas que comprometem a locomoção de um ou mais membros (Flower \& Weary, 2006). Em ambientes de criação intensiva, a ampliação e o confinamentos dos plantéis leiteiros devido ao avanço genético, nutricional e de manejo, levam a ocorrência das doenças podais por conta da concentração de animais em pisos ásperos e firmes (Molina et al., 1999; Pardo et al., 2004; Romani et al., 2004). Neste cenário, em muitos casos, a bovinocultura leiteira tem características essencialmente extrativistas, não só do ponto de vista econômico, mas também científico, porém, a necessidade de incrementar medidas de bem-estar animal para se garantir no comércio internacional, utilizando sistema de produção animal mais humanitário (Amaral \& Trevisan, 2017). As afecções digitais constituem uma das principais afecções do aparelho locomotor dos bovinos que resultam em problemas de saúde de ordem local ou geral com dificuldades de locomoção e claudicação (Silva et al., 2015). 
Os estudos clínico-cirúrgicos que abrangem as afecções digitais em bovinos leiteiros ainda são limitados, porém vem avançando com resultados promissores. No entanto, o controle e alívio da dor e do sofrimento associados a estas patologias ainda é um desafio na medicina bovina. Atenção especial da legislação das novas Leis de bem-estar tem sido considerada um ponto crucial. Observa-se que a sociedade apresenta-se mais sensível a essa questão, não abrangendo apenas os danos ou maus-tratos, mas sim o bem-estar real dos animais. Há a necessidade de políticas públicas de incentivo junto à sociedade para priorizar o bem-estar na cadeia de produção animal (Amaral \& Trevisan, 2017).

\section{Bem-estar e maus-tratos}

O bem-estar animal pode ser pode ser definido como o estado de um indivíduo em relação às suas tentativas em se adaptar ao meio em que vive, é considerado alto quando o indivíduo está em condições adequadas de saúde, conforto, nutrição e ambiente. Para expressar os comportamentos naturais os animais devem ser mantidos com boas práticas de manejo, sem medo, angústia ou dor (Broom \& Fraser, 2010; Broom \& Johnson, 1993).

A ética sugere que o papel fundamental para o médico veterinário na sociedade está relacionado direta ou indiretamente às ações de controle da dor e do sofrimento no uso de animais, fazendo valer o bem-estar animal. A avaliação da dor em bovinos representa um desafio e o seu tratamento é considerado inadequado em várias ocasiões, em decorrência da falta de conhecimento sobre percepção da dor nesta espécie, entre elas as advindas de afecções digitais (Nunes et al., 2021).

A dor e o sofrimento de bovinos leiteiros acometidos por podopatias têm sido considerados complexos e estão compreendidos por fatores que envolvem a interação dos animais, micro-organismos e o ambiente (Amaral \& Trevisan, 2017). Problemas de locomoção oriundos de doenças digitais associadas à dor e desconforto são comuns e na maioria das vezes apenas os casos graves de claudicação são tratados sendo os casos menos graves negligenciados (Molina et al., 1999; Romani et al., 2004).

No âmbito científico o bem-estar é classificado em graus que variam de muito baixo a muito alto. Quando o animal estiver em grau de bem-estar baixo ou muito baixo, sem que o responsável por ele apresente ações que demonstrem tentativas de evitar, solucionar ou pelo menos minimizar a dor e sofrimento animal, estamos diante de um diagnóstico de maus-tratos. Estes também podem ser definidos como ações diretas ou indiretas que apontam negligência, agressão ou qualquer forma de ameaça ao bem-estar do indivíduo (Hammerschimidt \& Molento, 2017).

A necessidade de proteger os animais e evitar crueldade, abuso e maus-tratos é notório no início de um novo mundo globalizado. De uma forma geral é comum qualquer tipo de lesão ser enquadrada como maus-tratos, no entanto, diagnósticos de maus-tratos é algo mais amplo e pode dificultar a compreensão da gravidade por parte da autoridade judicial (Tremori et al., 2018). No Brasil, os casos de maus-tratos aos animais estão sujeitos a sanções penais e administrativas através da Lei Federal $n^{\circ}$ 9605/98, denominada Lei de Crimes Ambientais. No Capítulo V, Art. 32 determina que: "praticar ato de abuso, maus-tratos, ferir ou mutilar animais silvestres, domésticos ou domesticados, nativos os exóticos. Penadetenção de três meses a um ano, e multa". Neste capítulo está definido: $\S 1^{\circ}$ "incorre nas mesmas penas quem realizar experiência dolorosa ou cruel em animal vivo, ainda que para fins didáticos ou científicos, quando existem recursos alternativos". No $\S 2^{\circ}$ define: "a pena é aumentada de um sexto a um terço, se incorre a morte do animal“" (BRASIL, 1988).

No Brasil a Resolução 1.236/2018, do Conselho Federal de Medicina Veterinária, considera que médicos veterinários apresentam competência legal para identificar, caracterizar e diagnosticar casos de crueldade, abuso e maus-tratos em animais, principalmente em casos que envolvam a perícia (BRASIL, 2018). Associações veterinárias e legisladores no mundo ainda não concordaram totalmente quanto às responsabilidades dos médicos veterinários em ações para defender e aliviar a dor e sofrimento animal, dando início a uma ação globalizada. Cada vez mais o papel do médico veterinário não se restringe apenas a tratar animais, mas em defender o bem-estar, sendo um comportamento relativamente recente, fundamentado em dados empíricos que fazem elos com o abuso e a violência interpessoal. Segundo a Associação Médica Veterinária Canadense os médicos veterinários têm "obrigação moral" de comunicar casos suspeitos de maus-tratos em animais, assim como, apoiarem outros médicos veterinários que comunicam casos de boa-fé usando seus julgamentos profissionais. Em 2010 a Associação Médica 
Veterinária Americana alterou o juramento veterinário para incluir a proteção e bem-estar animal e a prevenção do sofrimento animal (Arkow \& Nassaro, 2017).

\section{As ciências forenses e as perícias veterinárias}

As ciências forenses atuam no processo de desenvolvimento e transferência de conhecimento científico e tecnológico nas ciências naturais, com objetivo de aplica-los na avaliação de vestígios, para responder questões científicas de interesse da justiça (Santos-Filho \& Mayrink, 2017; Velho et al., 2017). A medicina legal está compreendida como importante ciência forense auxiliar, sendo responsável pela realização de perícias nas áreas de sua competência, que em alguns casos é indispensável no auxílio à justiça, atendendo as necessidades públicas e sociais (Costa \& Costa, 2015).

Cada vez mais a medicina veterinária legal vem se destacando no progresso e na relevância concedida aos animais e seus direitos (Slowinski et al., 2016). Esta área abrange, de forma direta ou indireta, os animais e seus subprodutos, saúde pública, defesa sanitária, defesa do consumidor, fauna, bem-estar animal e meio ambiente (Larsson \& Lucas, 2016; Marlet \& Maiorka, 2010; Reis, 2017; Tostes \& Reis, 2017). O termo perícia define a expertise e representa de prova relevante para atender a demanda do Poder Judiciário ou do Ministério Público, necessário nos processos civis e penais realizados por profissionais especializados e capacitados (Conceição et al., 2017).

Em tempos remotos os animais comumente eram alvos de tema forense em decorrência de roubos e danos indevidamente por terem valor de coisa, objetos de posse humana, sem o valor da dor e do sofrimento acometidos a eles. Na década de 60 foi publicado o livro Animal Machines, de autoria de Ruth Harrison, que evidenciou o sofrimento dos animais de produção, sendo um marco da era do bem-estar animal. Todavia, somente após a década de 90 ocorreram as primeiras divulgações e publicações de maustratos a animais nas revistas de criminologia. Todos os animais tutelados de humanos estão em riscos permanentes ou transitórios de maus-tratos, sendo a negligência a causa mais frequente e menos explorada cientificamente (Arkow \& Nassaro, 2017; Hammerschimidt \& Molento, 2017; Marlet \& Maiorka, 2010).

Com ampliação da informação da tutela jurídica, a demanda de perícias veterinárias vem crescendo devido ao aumento do número de processos penais envolvendo tanto animais como seus produtos (Arkow \& Nassaro, 2017; Hammerschimidt \& Molento, 2017). Uma crescente preocupação do homem com os animais e com o meio ambiente vem sendo destacada nos últimos 10 anos. Neste contexto, observou-se um aumento na demanda de perícias veterinárias, envolvendo tanto animais de companhia, animais silvestres, domesticados, quanto a animais de produção. As perícias visam ajudar na investigação e na elucidação de fatos em diferentes circunstâncias envolvendo o bem-estar animal e crimes de crueldade (Tremori et al., 2018; Tremori \& Rocha, 2013).

Do ponto de vista da clínica médica forense destaca-se o exame de corpo de delito para avaliação de um delito aplicando-se a exploração clínica minuciosa que fornece dados para elaboração de laudos para o auxílio e à condução de um processo judicial. O médico veterinário deve se conduzir pelo Código de Ética Médica que possa ser comprometido por imperícia, imprudência ou negligência no exercício profissional (Garcia, 2017; Maiorka, 2016). O exame de corpo de delito deve ser realizado por profissionais competentes e capacitados para exercer função de elaborar uma fundamentação de um determinado delito (Rocha, 2017). Na instância penal o exame de corpo de delito tem a finalidade de investigar os elementos que possam auxiliar na ocorrência de um delito (Guimarães, 2017; Santos-Filho \& Mayrink, 2017; Velho et al., 2017). Em muitos casos, as ocorrências de lesões causadas nos animais são frequentemente interpretadas como maus-tratos, no entanto, esta condição pode confundir o julgamento e a compreensão do magistrado, em decorrência da complexidade do diagnóstico e definição de maus-tratos (Tremori et al., 2018).

\section{Discussão}

As ciências forenses atuam no processo de desenvolvimento e transferência de conhecimento científico e tecnológico nas ciências naturais, com objetivo de aplicar na avaliação de vestígios, para responder questões científicas de interesse da justiça (Velho et al., 2017). Por ser considerada uma área interdisciplinar, as ciências forenses envolve várias áreas de conhecimento e seu objetivo é dar suporte às investigações relativas à justiça civil e criminal, tendo como foco principal confirmar ou descartar o 
envolvimento do suspeito. Neste contexto, a medicina veterinária legal tem campo de destaque nas áreas de competência da medicina veterinária, incluindo o bem-estar animal.

Do ponto de vista da clínica médica forense destaca-se o exame de corpo de delito para avaliação de um delito aplicando-se a exploração clínica minuciosa que fornece dados para elaboração de laudos para o auxílio e à condução de um processo judicial. O médico veterinário deve se conduzir pelo Código de Ética Médica que possa ser comprometido por imperícia, imprudência ou negligência no exercício profissional (Costa \& Schuchmann, 2018). O médico veterinário capacitado poderá atuar em diversas áreas da perícia e o bem-estar animal de animais de produção representa um campo promissor nas perícias veterinárias, destacando a patologia e a clínica médica forense nas revelações de diversos delitos por negligência, imperícias e imprudências em casos de maus-tratos, crueldade e abuso contra os animais.

O bem-estar animal pode ser pode ser definido como o estado de um indivíduo em relação às suas tentativas em se adaptar ao meio em que vive, é considerado alto quando o indivíduo está em condições adequadas de saúde, conforto, nutrição e ambiente. Para expressar os comportamentos naturais os animais devem ser mantidos com boas práticas de manejo, sem medo, angústia ou dor (Broom, 1986). O diagnóstico do bem-estar tem fundamentação na patologia e na clínica médica associada a avaliação do comportamento e exames complementares. Dessa forma, é importante a condução dos trabalhos de diagnóstico de bem-estar específico para animais envolvidos na produção de leite no Brasil. Os métodos de diagnósticos permitem evidenciar os principais pontos críticos do bem-estar de bovinos leiteiros. Permitem que sejam desenvolvidas técnicas de diagnóstico específicas para a realidade da produção de leite brasileira.

A dor e o sofrimento estão presentes em todos os sistemas de exploração de bovinos leiteiros e as afecções digitais, associadas ou não as claudicações, representam um dos mais importantes problemas de saúde que compromete o bem-estar desta categoria animal (Amaral \& Trevisan, 2017). Diante desta realidade produtores rurais e técnicos especializados enfrentam grande desafio para manter os sistemas de produção com manejo humanitário que atendam ás exigências de mercado nacional e internacional em padrões de saúde e bem-estar, seguindo os limites técnicos, éticos e legais.

Animais de produção são frequentemente manejados na rotina que causa dor ou desconforto em vários procedimentos como castração, descorna, auxílios obstétricos em distocias, sem aplicação prévia de anestesia e analgesia (Hudson et al., 2008). Os problemas de locomoção decorrentes de afecções digitais são frequentes e na rotina do manejo frequentemente é realizado apenas em casos graves de claudicação, havendo negligência no tratamento dos animais nos casos menos graves pelo simples fato de não serem diagnosticados ou por motivos econômicos. Diante desta realidade o diagnóstico de bemestar dos rebanhos tem fundamental importância no reconhecimento precoce dos animais acometidos por estas afecções. A identificação de animais com processos dolorosos nas fases iniciais tem importância fundamental na prevenção e utilização de anestésicos e analgésicos de forma racional, diminuindo custos e mão-de-obra nos tratamentos.

As podopatias constituem uma das principais afecções do aparelho locomotor dos bovinos que resultam em problemas de saúde de ordem local ou geral com dificuldades de locomoção e claudicação (Silva et al., 2015). As claudicações em bovinos estão frequentemente associadas a dor que produzem desordens biomecânicas que comprometem a locomoção de um ou mais membros (Flower \& Wear, 2006). Portanto, dietas ricas em carboidratos, falta de podologia preventiva dos cascos, manutenção de animais em ambientes úmidos e trânsitos ou manutenção de animais em terrenos pedregosos são alguns fatores de riscos que contribuem para a ocorrência de afecções digitais. Para garantir a produção os pecuaristas enfrentam problemas que comprometem o desempenho dos animais, sendo geralmente problemas de manejo, nutrição inadequada, instalações mal dimensionadas e cuidados na saúde geral e reprodutivo dos animais. Neste contexto, levam a ocorrência de afecções digitais que levam a dor, claudicação, complicações com mastite e distúrbios reprodutivos e posteriormente declínio na produção.

Observa-se que a sociedade estar mais sensível à questão da dor e do sofrimento animal e esta preocupação não abrange apenas a danos ou maus-tratos, mas sim ao bem-estar real dos animais. Há necessidade de políticas públicas de incentivo junto à sociedade para priorizar o bem-estar na cadeia de produção animal (Amaral \& Trevisan, 2017). 
A avaliação do bem-estar do ponto de vista científico deve ser considerada separada das questões éticas. As técnicas de diagnóstico dos graus de bem-estar fundamentado na clínica médica e exames complementares, são importantes e necessários para realização dos laudos de perícias de bem-estar. O Welfare quality (2009) auxilia na avaliação dos indicadores nutricionais, de conforto, sanitários e comportamentais, os quais são fundamentais para conclusão dos laudos periciais. Casos de maus-tratos, crueldade ou abuso por negligência imperícia e imprudência podem ser constatados pelas perícias de bem-estar e as penalidades podem ser enquadradas na Lei de Crimes Ambientais, $n^{\circ}$ 9.605/98 e na Resolução CFMV nº 1236/2018 (BRASIL, 2018).

\section{Conclusão}

O avanço do conhecimento no diagnóstico e tratamento das afecções digitais tem resultados promissores, no entanto, o controle da dor e sofrimento e a alta incidência em animais acometidos por estas afecções ainda continua um desafio. As afecções digitais causam dor, sofrimento e claudicação que comprometem a produção, com agravante da necessidade dos animais serem manejados na rotina nestas condições. As decisões judiciais em casos de maus-tratos podem ser subsidiadas por pareceres técnicos de especialistas em bem-estar animal. Casos de maus-tratos, crueldade ou abuso por negligência imperícia e imprudência podem ser constatados pelas perícias de bem-estar em laudos utilizados em questões judiciais.

\section{Referências bibliográficas}

Amaral, J. B., \& Trevisan, G. (2017). Aspectos da dor e sofrimento no bem-estar de bovinos leiteiros acometidos por podopatias. PUBVET, 11, 1074-1187. https://doi.org/10.22256/pubvet.v11n11.1074-1084.

Arkow, P., \& Nassaro, M. R. F. (2017). Maus-tratos a animais no contexto de outra violência familiar. In R. A. Tostes, Reis S. T. J., \& V. V Castilho (Eds.), Tratado de Medicina Veterinária Legal. Medvep.

BRASIL, Lei 9.605, de 12 de fevereiro de 1988, Lei de Crimes Ambientais: dispõe sobre as sanções penais e administrativas derivadas de condutas e atividades lesivas ao meio ambiente, e dá outras providências. http://www.planalto.gov.br/ccivil_03/LEIS/L9605.htm [Acesso em 22 de Mar de 2021].

BRASIL, Resolução 1.236, de 26 de outubro de 2018. Define e caracteriza crueldade, abuso e maus-tratos contra animais vertebrados, dispõe sobre a conduta de médicos veterinários e zootecnistas e dá outras providências. https://www.crmvpr.org.br/uploads/noticia/arquivos/reso-CFMV1236_2018.pdf [Acesso 22 de Mar de 2021].

Broom, D. M. (1986). Indicators of poor welfare. The Britsh Veterinary Journal, 142, 524-526. 1986

Broom, D M. (1991). Animal welfare: concepts and measurement. Journal of Animal Science, 69(10), 4167-4175.

Broom, Donald M, \& Fraser, A. F. (2010). Comportamento e bem-estar de animais domésticos. Editora Manole.

Broom, Donald M, \& Johnson, K. G. (1993). Stress and animal welfare. Springer Science \& Business Media.

Conceição, C. D. C., Almeida, E. C. P., \& Marcon, F. M. (2017). Novo código de ética médica veterinária - Comentários sobre a ótica pericial.

Costa, G. R., \& Schuchmann, R. K. (2018). Clínica forense: exame de corpo de delito (vítima com vida). In R. C. M. Garcia, Y. . Gonçalves, K. A. Rossa, \& L. R. Wof (Eds.), Tópicos em medicina veterinária legal. Universidade Federal do Paraná. Universidade Federal do Paraná.

Costa, L. R. S., \& Costa, B. M. C. (2015). A perícia médico-legal aplicada à área criminal. Ediitora Millennium.

Croce, D., \& Croce Júnior, D. (2017). Manual de medicina legal. Saraiva Educação SA.

Ferreira, P. M., Leite, R. C., Carvalho, A. U., Facury Filho, E. J., Souza, R. C., \& Ferreira, M. G. (2004). Custo e resultados do tratamento de seqüelas de laminite bovina: relato de 112 casos em vacas em lactação no sistema free-stall. Arquivo Brasileiro de Medicina Veterinária e Zootecnia, 56(5), 589-594. 
Flower, F. C., \& Weary, D. M. (2006). Effect of hoof pathologies on subjective assessments of dairy cow gait. Journal of Dairy Science, 89(1), 139-146. https://doi.org/http://dx.doi.org/10.3168/jds.S0022-0302(06)72077-X

França, G. V. (2005). Fundamentos de medicina legal. Guanabara Koogan S.A.

Garbarino, E. J., Hernandez, J. A., Shearer, J. K., Risco, C. A., \& Thatcher, W. W. (2004). Effect of lameness on ovarian activity in postpartum Holstein cows. Journal of Dairy Science, 87(12), 41234131. https://doi.org/10.3168/jds.S0022-0302(04)73555-9.

Garcia, R. C. M. (2017). Desafios para o enfrentamento da negligência. In R. A. Tostes, S. T. J. Reis, \& V. V Castilho (Eds.), Tratado de medicina veterinária legal. Medvep.

Guimarães, M. A. (2017). Medicina legal. In J. A. Velho, G. C. Geiser, \& A. Espindula (Eds.), Ciências Forenses: uma introdução às principais áreas da criminalística moderna. Millenium Editora.

Hammerschimidt, J., \& Molento, C. F. M. (2017). Perícia em bem-estar animal nos crimes de maustratos contra animais. In R. A. Tostes, S. T. J. Reis, \& V. V Castilho (Eds.), Tratado de medicina veterinária legal. Medvep.

Hudson, C., Whay, H., \& Huxley, J. (2008). Recognition and management of pain in cattle. In Practice, 30(3), 126-134. https://doi.org/10.1136/vr.f6253.

Koknaroglu, H., \& Akunal, T. (2013). Animal welfare: An animal science approach. Meat Science, 95(4), 821-827. https://doi.org/http://dx.doi.org/10.1016/j.meatsci.2013.04.030

Larsson, C. E., \& Lucas, R. (2016). Tratado de medicina externa: dermatologia veterinária. Interbook.

Lean, I. J., \& Golder, H. M. (2019). Ruminal acidosis: Beyond pH and rumen. 2019 Cornell Nutrition Conference for Feed Manufactures, 127-145.

Luna, S. P. L. (2006). Dor e sofrimento animal. In E. A. B. Rivera, M. H. Amaral, \& V. P. Nascimento (Eds.), Ética e bioética (pp. 131-158).

Maiorka, P. C. (2016). Medicina Veterinária Legal: uma demanda crescente da sociedade. Revista Do Conselho Regional Do Estado de São Paulo, 61, 10-18.

Marlet, E. F., \& Maiorka, P. C. (2010). Análise retrospectiva de casos de maus tratos contra cães e gatos na cidade de São Paulo. Brazilian Journal of Veterinary Research and Animal Science, 47(5), 385394. https://doi.org/10.11606/issn.1678-4456.bjvras.2010.26820.

Molina, L. R., Carvalho, A. U., Facury Filho, E. J., Ferreira, P. M., \& Ferreira, V. C. P. (1999). Prevalência e classificação das afecções podais em vacas lactantes na bacia leiteira de Belo Horizonte. Arquivo Brasileiro de Medicina Veterinária e Zootecnia, 51(2), 149-152.

Mülling, C. K. W., Green, L., Barker, Z., Scaife, J., Amory, J., \& Speijers, M. (2006). Risk factors associated with foot lameness in dairy cattle and a suggested approach for lameness reduction. World Buiatrics Congress, 24.

Nunes, M. H. V., Pacheco, A. D., \& Wagatsuma, J. T. (2021). Reconhecimento e avaliação da dor em bovinos: Revisão. PUBVET, 15(6), 1-12. https://doi.org/10.31533/pubvet.v15n06a831.1-12.

Ollhoff, R D, Pereira, I. R. A., Luz, N. C., \& Machado, F. G. (2005). Incidência de alterações dentárias em um rebanho bovino leiteiro. Archives of Veterinary Science, 10(2), 95-100. https://doi.org/10.5380/avs.v10i2.4423.

Ollhoff, Rüdiger Daniel, \& Ortolani, E. L. (2001). Comparação do crescimento e do desgaste do casco em bovinos taurinos e zebuínos. Ciência Rural, 31, 67-71. https://doi.org/10.1590/s010384782001000100011.

Pardo, P. E., Bremer Neto, H., Chiacchio, S. B., Nagoshi, M., \& Padilha, P. M. (2004). Determinação de zinco da sola do casco de bovinos leiteiros com ou sem lesões podais, suplementados ou não com levedura seca de cana-de-açúcar. Ciência Rural, 34, 1501-1504. https://doi.org/10.1590/s010384782004000500027.

Reis, S. T. J. (2017). Perícia de local de crime envolvendo animais - Parte I. In R. A. Tostes, S. T. J. Reis, \& V. V Castilho (Eds.), Tratado de medicina veterinária legal. Medvep.

Rocha, N. S. (2017). Corpo de delito e processos por erro médico veterinário. In R. A. Tostes, S. T. J. Reis, \& V. V Castilho (Eds.), Tratado de medicina veterinária legal. Medvep.

Romani, A. F., Silva, L. A. F. ., \& Fioravanti, M. C. S. (2004). Ocorrência de lesões podais em fêmeas 
bovinas leiteiras no Estado de Goiás. ARS Veterinaria, 20(3), 322-392.

Santos-Filho, A. M. P., \& Mayrink, R. R. (2017). Medicina Veterinária Forense. In J. A. Velho, G. C. Geiser, \& A. Espíndula A. (Eds.), Ciências Forenses, uma introdução às principais áreas da criminalística moderna. Millennium.

Silva, F. F., Alves, C. G. T., \& Silva Júnior, F. F. (2016). Pododermatite solar circunscrita, úlcera de husterholz ou úlcera da sola. Ciência Veterinária Nos Trópicos, 9(2/3), 102-105.

Silva, Luiz A F, Campos, S. B. S., Rabelo, R. E., Vulcani, V. A. S., Noronha Filho, A. D. F., \& Freitas, S. L. R. (2015). Análise comparativa da morfometria do casco de bovinos das raças Nelore, Curraleira e Pantaneira e de bubalinos e sua relação com a etiopatogenia das enfermidades digitais. Pesquisa Veterinária Brasileira, 35(4), 377-384.

Silva, Luiz Antônio Franco, Rezende, M. R., Romani, A. F., Fioravanti, M. C. S., Cunha, P. H. J., Borges, J. R. J., Macedo, S. P., Damasceno, A. D., Rabelo, R. E., \& Garcia, A. M. (2006). Pododermatite séptica em bovinos: evolução clínica da fase inicial. Brazilian Journal of Veterinary Research and Animal Science, 43(5), 674-680.

Slowinski, K., Tremori, T. M., Massad, M. R. R., Tasaka, A. C., \& Rocha, N. S. (2016). Responsabilidade ética e civil do médico-veterinário no ambiente hospitalar. Revista de Educação Continuada Em Medicina Veterinária e Zootecnia Do CRMV-SP, 14(2), 30-37.

Souza, R. C., Ferreira, P. M., Molina, L. R., Carvalho, A. U., \& Facury Filho, E. J. (2006). Perdas econômicas ocasionadas pelas enfermidades podais em vacas leiteiras confinadas em sistema free stall. Arquivo Brasileiro de Medicina Veterinária e Zootecnia, 58, 982-987. https://doi.org/10.1590/S0102-09352006000600002.

Tostes, R. A., \& Reis, S. T. J. (2017). História da medicina veterinária legal - Parte II. In R. A. Tostes, S. T. J. Reis, \& V. V Castilho (Eds.), Tratado de medicina veterinária legal. Medvep.

Tremori, T. M., Ribas, L. M., Massad, M. R. R., Reis, S. T. J., Pinto, A. C. F., \& Rocha, N. S. (2018). Classificação comparada das lesões de ordem mecânica segundo a traumatologia forense no exame de corpo de delito em animais. Revista Brasileira de Criminalística, 7(2), 20-25.

Tremori, T. M., \& Rocha, N. S. (2013). Exame do corpo de delito na Perícia Veterinária (ensaio). Revista de Educação Continuada Em Medicina Veterinária e Zootecnia Do CRMV-SP, 11(3), 30-35.

Velho, J. A., Geiser, G. C., \& Espindula, A. (2017). Ciências Forenses: Uma introdução às principais áreas da criminalística moderna. Millenium Editora.

Welfare Quality (2009). Welfare Quality assessment protocol for cattle. Consortium, Lelystad, Netherlands.

Histórico do artigo:

Recebido: 15 de agosto de 2021

Aprovado: 17 de setembro de 2021

Disponível online: 18 de novembro de 2021
Licenciamento: Este artigo é publicado na modalidade Acesso Aberto sob a licença Creative Commons Atribuição 4.0 (CC-BY 4.0), a qual permite uso irrestrito, distribuição, reprodução em qualquer meio, desde que o autor e a fonte sejam devidamente creditados. 